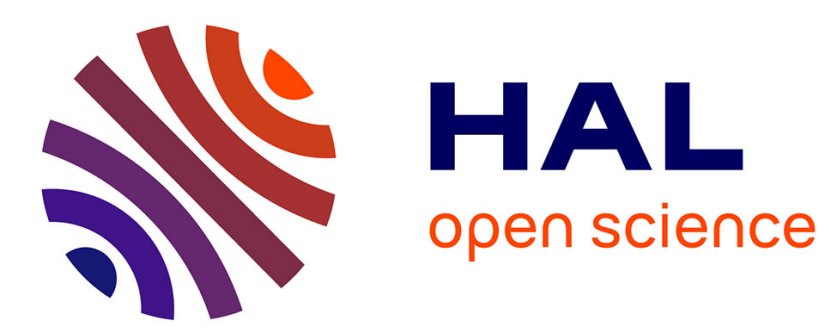

\title{
Using Logarithmic Penalties in the Shooting Algorithm for Optimal Control Problems
}

\author{
J. Frederic Bonnans, Thérèse Guilbaud
}

\section{To cite this version:}

J. Frederic Bonnans, Thérèse Guilbaud. Using Logarithmic Penalties in the Shooting Algorithm for Optimal Control Problems. [Research Report] RR-4237, INRIA. 2001. inria-00072350

\section{HAL Id: inria-00072350 \\ https://hal.inria.fr/inria-00072350}

Submitted on 23 May 2006

HAL is a multi-disciplinary open access archive for the deposit and dissemination of scientific research documents, whether they are published or not. The documents may come from teaching and research institutions in France or abroad, or from public or private research centers.
L'archive ouverte pluridisciplinaire HAL, est destinée au dépôt et à la diffusion de documents scientifiques de niveau recherche, publiés ou non, émanant des établissements d'enseignement et de recherche français ou étrangers, des laboratoires publics ou privés. 
INSTITUT NATIONAL DE RECHERCHE EN INFORMATIQUE ET EN AUTOMATIQUE

\section{Using logarithmic penalties in the shooting algorithm for optimal control problems}

J. Frédéric Bonnans — Thérèse Guilbaud

$\mathbf{N}^{\circ} \mathbf{4 2 3 7}$

Septembre 2001

THÈME 4 



\title{
Using logarithmic penalties in the shooting algorithm for optimal control problems
}

\author{
J. Frédéric Bonnans*, Thérèse Guilbaud ${ }^{\dagger}$ \\ Thème 4 - Simulation et optimisation \\ de systèmes complexes \\ Projet SYDOCO \\ Rapport de recherche $n^{\circ} 4237$ - Septembre 2001 - 23 pages
}

\begin{abstract}
The paper deals with optimal control problems of ordinary differential equations with bound control constraints. We analyse the logarithmic penalty method for converting the problem into an unconstrained one, the latter being solved by a shooting algorithm. Convergence of the value function and optimal controls is obtained for linear quadratic problems, and more generally when the control variable enters linearly in the state equation and in a quadratic way in the cost function. We display some numerical results on two examples: an aircraft maneuvre, and the stabilization of an oscillating system.
\end{abstract}

Key-words: Optimal control, logarithmic penalty, shooting algorithms, interior point methods, perturbed optimization, flight mechanics, linear oscillator

* INRIA, B.P. 105, 78153 Rocquencourt, France. Email: Frederic.Bonnans@inria.fr.

$\dagger$ INRIA, B.P. 105, 78153 Rocquencourt, France. Email: Therese.Guilbaud@inria.fr. 


\section{Sur l'utilisation de pénalités logarithmiques dans l'algorithme de tir pour la commande optimale}

Résumé : Cet article traite les problèmes de commande optimale d'équations différentielles avec contraintes de borne sur la commande. Nous analysons la méthode de pénalisation logarithmique, qui convertit le problème en un problème sans contraintes, ce dernier étant résolu par un algorithme de tir. Nous obtenons la convergence de la fonction valeur et des commandes optimales pour les problèmes linéaires quadratiques, et plus généralement quand la commande intervient linéairement dans l'équation d'état et de manière quadratique dans la fonction coût. Nous donnons des résultats numériques dans deux cas: une manœuvre aérienne, et la stabilisation d'un système oscillant.

Mots-clés : Commande optimale, pénalisation logarithmique, algorithme de tir, méthodes de points intérieurs, optimisation avec perturbation, mécanique du vol, oscillateur linéaire 


\section{Introduction}

This paper deals with optimal control problems of the following form:

$$
\left\{\begin{array}{l}
\underset{u}{\operatorname{Min}} J(y, u):=\int_{0}^{T} \ell(y(t), u(t)) d t+\Phi(y(T)) \\
\left\{\begin{array}{l}
\dot{y}(t)= \\
y(0)=y_{0}
\end{array}\right. \\
u \in U_{a d} .
\end{array}\right.
$$

Here the horizon $T>0$ and initial state $y_{0} \in \mathbb{R}^{n}$ are given, while the distributed and final cost function $\ell: \mathbb{R}^{n} \times \mathbb{R}^{m} \rightarrow \mathbb{R}$ and $\Phi: \mathbb{R}^{n} \rightarrow \mathbb{R}$, as well as the dynamics $f: \mathbb{R}^{n} \times \mathbb{R}^{m} \rightarrow \mathbb{R}^{n}$, satisfy appropriate assumptions that will be specified later. The set of feasible controls is

$$
U_{a d}:=L^{2}(0, T ; U)
$$

where

$$
U=\left\{u \in R^{m} / a_{i} \leq u_{i} \leq b_{i} i=1, \ldots, m\right\},
$$

and $a_{i}, b_{i} \in \overline{\mathbb{R}}=\mathbb{R} \cup\{+\infty,-\infty\}$ are given extended real numbers. With this problem is associated the Hamiltonian

$$
H(y, u, p)=\ell(y, u)+p^{T} f(y, u) .
$$

A basic idea for solving this problem is to use Pontryaguin's Maximum Principle. The latter states that a necessary condition for $(y, u)$ to be a solution of $(P)$ is the existence of an adjoint state $p$ such that

$$
\left\{\begin{array}{l}
\dot{y}(t)=H_{p}(y(t), u(t), p(t)), \quad t \in[0, T], \\
\dot{p}(t)=-H_{y}(y(t), u(t), p(t)), \quad t \in[0, T], \\
y(0)=y_{0} ; \quad p(T)=\Phi^{\prime}(y(T)), \\
u(t) \in \underset{v \in U}{\operatorname{Argmin}} H(y(t), p(t), v), \quad t \in[0, T] .
\end{array}\right.
$$

Assume that we may eliminate the control variable from the last relation, i.e., that this relation is equivalent to $u(t)=\Upsilon(y(t), p(t))$, for some function $\Upsilon: \mathbb{R}^{n} \times \mathbb{R}^{n} \longrightarrow \mathbb{R}$. Then the above system is equivalent to the differential system with differential variables $(y, p)$

$$
\begin{cases}\dot{y}(t)=H_{p}(y(t), \Upsilon(y(t), p(t)), p(t)), & t \in[0, T], \\ \dot{p}(t)=-H_{y}(y(t), \Upsilon(y(t), p(t)), p(t)), \quad t \in[0, T], & \\ y(0)=y_{0} ; \quad p(T)=\Phi^{\prime}(y(T)) . & \end{cases}
$$

$\mathrm{RR} \mathrm{n}^{\circ} 4237$ 
This is a two points boundary value problem. A standard technique for solving it is the shooting method (see Stoer and Bulirsch [16]) that we present now. For a given value $p_{0}$ of the adjoint state at time 0 , under appropriate technical conditions, the following Cauchy problem is well posed:

$$
\begin{cases}\dot{y}(t)=H_{p}(y(t), \Upsilon(y(t), p(t)), p(t)), & t \in[0, T], \\ \dot{p}(t)=-H_{y}(y(t), \Upsilon(y(t), p(t)), p(t)), & t \in[0, T], \\ y(0)=y_{0} ; \quad p(0)=p_{0} . & \end{cases}
$$

Denoting as $y\left(t, p_{0}\right)$ and $p\left(t, p_{0}\right)$ the solution, we see that Pontryaguin's Maximum Principle is equivalent to finding a zero of the function $\Psi: \mathbb{R}^{n} \longrightarrow \mathbb{R}^{n}$, defined by

$$
\Psi\left(p_{0}\right):=p\left(T, p_{0}\right)-\Phi^{\prime}\left(y\left(T, p_{0}\right)\right) .
$$

Even if the data $\ell, f$, and $\Phi$ are smooth, and the Hamiltonian is a strongly convex function of the control, this function $\Psi$ is, in general, nondifferentiable. The reason is that, due to the bound constraints, $\Upsilon$ itself is nonsmooth. Therefore we cannot use Newton's method in a standard way for finding a zero of $\Psi$. Note that it might be of interest to consider the application to this problem of some nonsmooth variants of Newtons' method, see Martínez and Qi [13] and Chen et al. [8]).

The usual remedy when using shooting methods is to determine the arcs where the constraints become active or inactive and to split the optimality system in as many algebraic and differential equations as arcs, on which the algebraic constraint is an equality, then solvable with shooting algorithms (see Pesch [14]). This may be very heavy and requires information about the optimal solution. An extensive comparison between different numerical schemes was done in Diekhoff et al. [9]. A useful variant of the method, in which the optimal control problem is reduced to an optimization problem, whose parameters are either the initial costate $p_{0}$ or the control and some of its derivatives at time 0 , is presented in Dixon and Bartholomew-Biggs [10] and extended in Fraser-Andrews [12] to state constrained problems.

The shooting algorithms are often opposed to the direct method, i.e., the use of a nonlinear programming method for solving a discrete time version of the problem; some large scale implementations of the direct method are discussed in Betts and Huffman [2] and Bonnans and Launay [4]. A comparison between the two approaches and their possible combinations can be found in Bulirsch et al. [7], and Betts [1].

The alternative method we propose for dealing with constraints is based on the logarithmic penalty for dealing with the bound constraints. This is a classical idea (see Fiacco et McCormick [11]), on which the attention has been focused during the last decade, due to the large success of interior point methods, see e.g. [3, 17, 18]. The penalized problem, assuming all components of the vectors $a$ and $b$ to be finite, consists in minimizing the cost function

$$
J_{\varepsilon}(y, u)=J(y, u)-\varepsilon \sum_{i=1}^{m} \int_{0}^{T}\left[\log \left(u_{i}(t)-a_{i}\right)+\log \left(b_{i}-u_{i}(t)\right)\right] d t,
$$


with $\varepsilon>0$ and $J$ is the cost function of the initial problem. In this paper, we establish convergence results, and discuss the numerical resolution of the penalized problem by the shooting algorithm. In section 2 , we show the convergence of optimal value and solutions of the penalized problem to those of the original control problem. In addition, we show that the solution of the penalized problem remains far from its bound, the distance being at least of order of $\varepsilon$. These results are extended in section 3 to a specific class of nonlinear control problems, namely when the control enters linearly in the state equation and in a quadratic way in the cost function. Finally, in section 4, we present some numerical results for two problems: an aircraft manoeuvre, and the stabilization of an oscillating system.

\section{Linear quadratic case.}

\subsection{Problem statement and notations}

Consider the following state equation

$$
\left\{\begin{array}{l}
\dot{y}(t)=A(t) y(t)+B(t) u(t) \quad t \in[0, T] \\
y(0)=y_{0}
\end{array}\right.
$$

and associated optimal control problem:

$$
\left\{\begin{array}{l}
\operatorname{Min}_{u} J(y, u)=\frac{1}{2} \int_{0}^{T}\|y(t)-\tau(t)\|^{2} d t+\frac{N}{2} \int_{0}^{T}\|u(t)-\hat{u}(t)\|^{2} d t \\
\text { subject to (2) and } u \in U_{a d} .
\end{array}\right.
$$

Here $y(t)$ and $u(t)$ belong to $\mathbb{R}^{n}$ and $\mathbb{R}^{m}$, resp., $\|\cdot\|$ denotes the Euclidean norm, and the data of the problem are the weight of the control $N>0$, the horizon $T>0$, the dynamics $A(t)$ and $B(t)$, matrices of appropriate size, continuous functions of time, and the target $\tau \in L^{\infty}\left(0, T, \mathbb{R}^{n}\right)$ as well as the reference control $\hat{u} \in L^{\infty}\left(0, T, \mathbb{R}^{n}\right)$. The feasible domain, that takes into account the bound constraints on the control, was defined in (1).

Denote by $y_{u}$ the state $y$ associated to the control $u$, solution of the differential equation (2). The following well known results will be useful later on.

Proposition 1 For every $s \in\left[1,+\infty\left[\right.\right.$ and $u \in L^{s}\left(0, T ; \mathbb{R}^{m}\right)$, there exists an unique $y_{u} \in$ $W^{1, s}\left(0, T ; \mathbb{R}^{n}\right)$, solution of the differential equation (2), and the mapping $u \mapsto y_{u}$ is strongly continuous. Therefore,

(i) There exists $C>0$ such that, for all $v \in L^{2}\left(0, T ; \mathbb{R}^{m}\right)$, one has $\left\|y_{v}\right\|_{\infty} \leq C\left(\left\|y_{0}\right\|+\|v\|_{L^{1}\left(0, T ; \mathbb{R}^{m}\right)}\right)$.

(ii) The mapping $u \mapsto y_{u}$ is continuous from $L^{s}\left(0, T ; \mathbb{R}^{m}\right)$ to $W^{1, s}\left(0, T ; \mathbb{R}^{n}\right)$, both spaces being endowed with the weak topology. 
Problem $(P)$ can be rewritten as

$$
\min _{u \in U_{a d}} F(u)
$$

where $F(u)=J\left(y_{u}, u\right)$. Thanks to proposition 1 , the quadratic and strongly convex function $F$ is continuous, hence weakly lower semi continuous (w.l.s.c.). The feasible domain $U_{a d}$ being convex, closed and nonempty, we obtain that problem $(P)$ has a unique solution $u^{*}$. Let us state the first order optimality condition. The Hamiltonian function of the system is

$$
H(u, y, p, t):=\frac{1}{2}\|y-\tau(t)\|^{2}+\frac{N}{2}\|u-\hat{u}(t)\|^{2}+p^{T}(A y+B u) .
$$

We have that the control $u$ and associated state $y$ are solution of problem $(P)$ iff there exists an adjoint state $p \in H^{1}\left(0, T, \mathbb{R}^{n}\right)$ such that the following first order optimality system holds:

$$
\left\{\begin{array}{clll}
\dot{y}(t) & = & H_{p}(u(t), y(t), p(t), t), & y(0)=y_{0}, \\
\dot{p}(t) & = & -H_{y}(u(t), y(t), p(t), t), & p(T)=0 \\
H_{u_{i}}(u(t), y(t), p(t), t) & = & 0 \text { if } a_{i}<u_{i}(t)<b_{i}, & \\
H_{u_{i}}(u(t), y(t), p(t), t) & \geq 0 \text { if } u_{i}(t)=a_{i}, & \\
H_{u_{i}}(u(t), y(t), p(t), t) & \leq 0 & \text { if } u_{i}(t)=b_{i} .
\end{array}\right.
$$
$(P)$.

In the next section we study the family of logarithmic penalty problems associated with

\subsection{Logarithmically penalized problems}

Penalty problems are stated with new cost function defined for every $\varepsilon>0$, by

$$
F_{\varepsilon}(u):=F(u)-\varepsilon \sum_{i=1}^{m} \int_{0}^{T} \log \left(u_{i}(t)-a_{i}\right)+\log \left(b_{i}-u_{i}(t)\right) d t,
$$

where it must be understood that if some bound is infinite, then the corresponding term evaluates to 0 . Then we state a family of unconstrained problem,

$$
\operatorname{Min}_{u \in U_{a d}} F_{\varepsilon}(u) .
$$

In this section, we show that problems $\left(P_{\varepsilon}\right)$ do approximate $(P)$, in the sense that solutions of problems $\left(P_{\varepsilon}\right)$ strongly converge to the solution of $(P)$, as well as the state and costate that are solution of the perturbated optimality system, and the value function.

The first step is to insure that problem $\left(P_{\varepsilon}\right)$ has a solution, at least for small $\varepsilon>0$, and in addition, that this solution is interior to the feasible domain $U_{a d}$. A difficulty appears because of the infinite dimension. For finite dimensional problems, the logarithmic penalty is a barrier, in the sense that it prevents the solution from touching the boundary of the 
feasible domain, which insures that the solutions are strictly included in the feasible domain. In our case, there clearly exist controls such that

$$
\exists i, \quad \operatorname{infess} u_{i}=a_{i} \text { or } \operatorname{supess} u_{i}=b_{i}
$$

while both integrals $\int_{0}^{T} \log \left(u_{i}(t)-a_{i}\right) d t$ and $\int_{0}^{T} \log \left(b_{i}-u_{i}(t)\right) d t$ are well defined and finite. Denote by $\hat{U}_{a d}$ the domain of $F_{\varepsilon}$ (that does not depend on $\varepsilon$ ), that is to say

$$
\hat{U}_{a d}=\left\{u \in L^{2}\left(0, T ; \mathbb{R}^{m}\right) ; \log \left(u_{i}(t)-a_{i}\right) \in L^{1}(0, T) ; \log \left(b_{i}-u_{i}(t)\right) \in L^{1}(0, T)\right\} .
$$

Since the logarithm is a concave function, $\hat{U}_{a d}$ is a convex set, over which $F_{\varepsilon}$ is strongly convex. For $\alpha>0$, consider the family of subsets of $\hat{U}_{a d}$ :

$$
U_{\alpha}=\left\{u \in L^{2}\left(0, T ; \mathbb{R}^{m}\right), a+\alpha \leq u \leq b-\alpha \text { a.e }\right\} .
$$

Each of these sets is closed and convex. Given $\alpha>0$ and $u \in U_{\alpha}$, we have that the integrals

$$
\int_{0}^{T} \log \left(u_{i}(t)-a_{i}\right) d t, \quad \int_{0}^{T} \log \left(b_{i}-u_{i}(t)\right) d t
$$

are well defined and finite, since the logarithm function is sublinear over $[\alpha, \infty)$. On the other hand, for every $\alpha>0, F_{\varepsilon}$ is w.l.s.c. continuous over $U_{\alpha}$. The following lower estimate of $F_{\varepsilon}(u)$ will be useful later.

Lemma 1 There exists $C>0$ such that, for all $\varepsilon>0$ and $u \in \hat{U}_{a d}$, the following inequality holds:

$$
F_{\varepsilon}(u) \geq\left(1-\frac{2 \varepsilon}{N}\right) F(u)-C \varepsilon .
$$

Proof. It suffices to give the proof for the case when $m=1$, and both $a$ and $b$ are finite. For every $u \in U_{a d}^{\varepsilon}$, we have:

$$
\begin{aligned}
F_{\varepsilon}(u)= & \frac{1}{2} \int_{0}^{T}\left\|y_{u}(t)-\tau(t)\right\|^{2}+\frac{N}{2} \int_{0}^{T}\|u(t)-\hat{u}(t)\|^{2} \\
& -\varepsilon \int_{0}^{T} \log (u(t)-a) d t-\varepsilon \int_{0}^{T} \log (b-u(t)) d t \\
= & \frac{1}{2} \int_{0}^{T}\left\|y_{u}(t)-\tau(t)\right\|^{2}+\frac{1}{2}(N-2 \varepsilon) \int_{0}^{T}\|u(t)-\hat{u}(t)\|^{2}+ \\
& +\varepsilon \int_{0}^{T}\left[(u(t)-\hat{u}(t))^{2}-\log (u(t)-a)-\log (b-u(t))\right] d t .
\end{aligned}
$$

Let $\beta:=\|\hat{u}\|_{\infty}$. The function: $[a, b] \times[-\beta, \beta] \rightarrow \mathbb{R},(x, y) \mapsto(x-y)^{2}-\log (x-a)-\log (b-x)$ being lower bounded, say by $-m^{\prime}$, setting $m:=T m^{\prime}$, we obtain

$$
\begin{aligned}
F_{\varepsilon}(u) & \geq \frac{1}{2} \int_{0}^{T}\left\|y_{u}(t)-\tau(t)\right\|^{2} d t+\frac{N}{2}\left(1-\frac{2 \varepsilon}{N}\right) \int_{0}^{T}\|u(t)-\hat{u}(t)\|^{2} d t \\
& \geq\left(1-\frac{2 \varepsilon}{N}\right) F(u)-m \varepsilon .
\end{aligned}
$$

$\mathrm{RR} \mathrm{n}^{\circ} 4237$ 
We can now prove that, for every $\varepsilon>0$, problem $\left(P_{\varepsilon}\right)$ has a unique solution that belongs to a set $U_{\alpha}$.

Theorem 1 Let $\varepsilon>0$. Then problem $\left(P_{\varepsilon}\right)$ has a unique minimizer $u_{\varepsilon}^{*}$. Moreover, there exist $k>0$ and a function $\alpha(\varepsilon)>0$ such that

$$
\alpha(\varepsilon) \geq k^{-1} \varepsilon,
$$

and

$$
a+\alpha(\varepsilon) \leq u_{\varepsilon}^{*} \leq b-\alpha(\varepsilon), \text { a.e. } t \in(0, T) .
$$

Proof. Uniqueness of the solution follows from the strong convexity of the cost function $F_{\varepsilon}$ over its domain $\hat{U}_{a d}$. Let $\bar{u}$ be such that

$$
a<\operatorname{infess} \bar{u} \leq \operatorname{supess} \bar{u}<b .
$$

Then $\bar{u}$ is feasible for $\left(P_{\varepsilon}\right)$, and $F_{\varepsilon}(\bar{u}) \longrightarrow F(\bar{u})$ when $\varepsilon \downarrow 0$. It follows that

$$
\lim \sup \operatorname{val}\left(P_{\varepsilon}\right) \leq \operatorname{val}(P) .
$$

Combining with (3), we obtain that the set

$$
\hat{U}_{a d}^{1}:=\left\{u \in \hat{U}_{a d} ; \quad F_{\varepsilon}(u) \leq \operatorname{val}\left(P_{\varepsilon}\right)+1\right\}
$$

is bounded.

It remains to prove the existence of the solution, as well as (4)-(5). It is enough to give the proof when $u$ is scalar and $a=0, b=\infty$. Let $u \in U_{a d}^{1}$ be such that infess $u=0$. Given $\alpha>0$, define a perturbation of $u$, denoted by $v$, as follows:

$$
v(t)=\left\{\begin{array}{l}
u(t) \quad \text { if } u(t) \geq \alpha \\
2 \alpha \quad \text { otherwise. }
\end{array}\right.
$$

We will show that, when $\alpha \leq \alpha(\varepsilon)$, then $F_{\varepsilon}(v) \leq F_{\varepsilon}(u)$. We have

$$
F_{\varepsilon}(v)-F_{\varepsilon}(u)=\frac{1}{2} \Delta_{1}+\frac{N}{2} \Delta_{2}+\varepsilon \Delta_{3}
$$

where

$$
\begin{aligned}
\Delta_{1} & :=\int_{0}^{T}\left(\left\|y_{v}(t)-\tau(t)\right\|^{2}-\left\|y_{u}(t)-\tau(t)\right\|^{2}\right) d t, \\
\Delta_{2}: & =\int_{0}^{T}\left(|u(t)-\hat{u}(t)|^{2}-|v(t)-\hat{u}(t)|^{2}\right) d t, \\
\Delta_{3} & :=\int_{0}^{T} \log (u(t) / v(t)) d t .
\end{aligned}
$$

INRIA 
Let us give some upper estimates of each of these three terms. Since $u$ and $v$ are equal except on the set $\{u \leq \alpha\}$, we easily obtain that

$$
\Delta_{3}=\int_{\{u \leq \alpha\}} \log (u(t) / v(t)) d t \leq-\operatorname{mes}(u \leq \alpha) \log 2 .
$$

Similarly, denoting again $\beta:=\|\hat{u}\|_{\infty}$, we have

$$
\begin{aligned}
\Delta_{2} & =\int_{\{u \leq \alpha\}}\left(|v(t)-\hat{u}(t)|^{2}-|u(t)-\hat{u}(t)|^{2}\right) d t \\
& \leq \sup _{t \in\{u \leq \alpha\}}(|v(t)|+|u(t)|+2 \beta) \int_{\{u \leq \alpha\}}(|v(t)|-|u(t)|) d t \\
& \leq 2 \alpha(3 \alpha+2 \beta) \operatorname{mes}(u \leq \alpha) .
\end{aligned}
$$

As for $\Delta_{1}$, we get

$$
\begin{aligned}
\Delta_{1} & =\int_{0}^{T}\left(y_{u}(t)+y_{v}(t)-2 \tau(t)\right)^{T}\left(y_{u}(t)-y_{v}(t)\right) d t \\
& \leq\left\|y_{v}+y_{u}-2 \tau\right\|_{\infty} \int_{0}^{T}\left\|y_{u}(t)-y_{v}(t)\right\| d t .
\end{aligned}
$$

Since the set $U_{a d}^{1}$ is a bounded subset of and contains $u$, the set of all possible $v$ is bounded in $L^{2}\left(0, T, \mathbb{R}^{m}\right)$. Since $\tau \in L^{\infty}\left(0, T ; \mathbb{R}^{n}\right)$, by proposition 1 , there exists $K>0$ such that

$$
\left\|y_{v}+y_{u}-2 \tau\right\|_{\infty} \leq K
$$

Thanks again to proposition 1 , there exists a constant $C$ such that

$$
\int_{0}^{T}\left\|y_{u}(t)-y_{v}(t)\right\| d t \leq C \int_{0}^{T}\|u(t)-v(t)\| d t
$$

Therefore, there exists $c>0$ such that, for any $u$ in $U_{a d}^{1}$ and associated $v$,

$$
\Delta_{1} \leq c \alpha \operatorname{mes}(u \leq \alpha) \text {. }
$$

Finally, we obtain

$$
F_{\varepsilon}(v)-F_{\varepsilon}(u) \leq\left(\frac{1}{2} c \alpha+N \alpha(3 \alpha+2 \beta)-\epsilon \log 2\right) \operatorname{mes}(u \leq \alpha) .
$$

Let $\gamma>0$ be such that

$$
\left(\frac{1}{2} c+2 \beta N\right) \gamma<\log 2,
$$

and let $\alpha=\alpha(\varepsilon):=\gamma \varepsilon$. Then for $\varepsilon>0$ small enough, we have that

$$
\frac{1}{2} c \alpha(\varepsilon)+N \alpha(\varepsilon)(3 \alpha(\varepsilon)+2 \beta)<\epsilon \log 2,
$$


and hence, by (10), $F_{\varepsilon}(v) \leq F_{\varepsilon}\left(u^{*}\right)$, while $v \geq \bar{\alpha}>0$ a.e. This shows that

$$
\inf _{U_{a d}} F_{\varepsilon}=\inf _{U_{\alpha}} F_{\varepsilon} .
$$

This holds in particular, when $\varepsilon$ is small enough, when

$$
\alpha(\varepsilon):=\varepsilon c^{-1} \log 2 .
$$

Now let $\left(u_{k}\right)_{k \in \mathbb{N}}$ be a minimizing sequence of problem $\left(P_{\varepsilon}\right)$, hence (for large enough $k$ ) the sequence is included in $U_{a d}^{1}$. Using the above construction, we can build a sequence $\left(v_{k}\right)$ in $U_{\alpha}(\varepsilon)$. Since the strongly convex function $F_{\varepsilon}$ is upper bounded, and hence continuous on $U_{\alpha}(\varepsilon)$, using standard arguments, we have that it has a unique minimum $u_{\varepsilon}$, over $U_{\alpha}(\varepsilon)$. and hence, $F_{\varepsilon}$ attains its minimum over $\hat{U}_{a d}$ at the point $u_{\varepsilon}$.

Remark 1 The function $F_{\varepsilon}$ is strongly convex on the set $U_{a d}$, but is nowhere continuous. Therefore, the standard argument for existence of a minimum does not apply.

Remark 2 The lower bound on $\alpha$ obtained in theorem 2 is optimal. Indeed, consider the simple following example:

$$
\min _{u \geq 0} \frac{1}{2}\left\|u-u_{d}\right\|^{2}
$$

with $u \in L^{2}(-1,+1)$ and $u_{d}(t)=t, \forall t \in[-1,+1]$. Here we have a control and non state, but this problem still is of the kind considered here (we may add a dummy state equation with a zero solution, and a null target). The solution is $u_{0}(t)=t 1_{t \geq 0}$. The expression of the penalized problem is

$$
\min \frac{1}{2}\left\|u-u_{d}\right\|^{2}-\varepsilon \int_{-1}^{+1} \log (u(t)) d t
$$

and the unique solution is

$$
u_{\varepsilon}(t)=\frac{t+\sqrt{t^{2}+4 \varepsilon}}{2} .
$$

Its minimal value is $u_{\varepsilon}(-1)=\frac{\varepsilon}{2(1+\sqrt{1+\varepsilon})}=O(\varepsilon)$.

The next step is to obtain convergence results, defining in which way problems $\left(P_{\varepsilon}\right)$ are approximations for $(P)$. This is collected in the following statement.

Proposition 2 When $\varepsilon \downarrow 0$, the following relations hold:

(i) Convergence of optimal controls: $u_{\varepsilon}^{*} \longrightarrow u^{*}$ in $L^{2}\left(0, T ; \mathbb{R}^{m}\right)$.

(ii) Convergence of cost functions: $F_{\varepsilon}\left(u_{\varepsilon}^{*}\right) \longrightarrow F\left(u^{*}\right)$. 


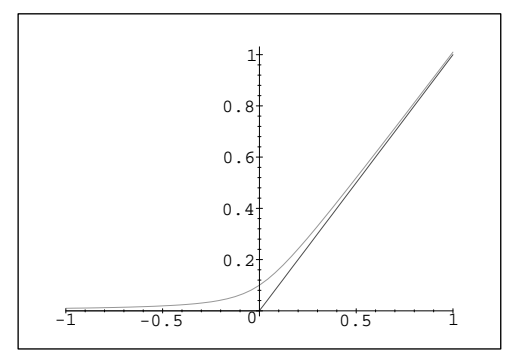

Figure 1: Functions $u_{0}$ and $u_{\varepsilon}$, with here $\varepsilon=10^{-3}$.

(iii) Convergence of states and costates associated with $u_{\varepsilon}^{*}$, denoted by $\left(y_{\varepsilon}^{*}\right)$ and $\left(p_{\varepsilon}^{*}\right)$, resp. $: y_{\varepsilon}^{*} \longrightarrow y^{*}$ and $p_{\varepsilon}^{*} \longrightarrow p^{*}$ uniformly on $[0, T]$.

Proof. Again, we may suppose that $m=1, a=0$ and $b=+\infty$. The first step is to show that $\lim \sup F_{\varepsilon}\left(u_{\varepsilon}^{*}\right) \leq F\left(u^{*}\right)$. Let $u_{0}$ satisfy $\operatorname{infess}\left(u_{0}\right)>0$. Given $\left.\eta \in\right] 0,1\left[\right.$, set $u_{\eta}:=$ $\eta u^{*}+(1-\eta) u_{0}$. Then $\operatorname{infess}\left(u_{\eta}\right)>0$ and $u_{\eta}$ is feasible for $\left(P_{\varepsilon}\right)$. Since $u_{\varepsilon}^{*}$ is optimal for $\left(P_{\varepsilon}\right)$, we have that

$$
F_{\varepsilon}\left(u_{\varepsilon}^{*}\right) \leq F_{\varepsilon}\left(u_{\eta}\right)=F\left(u_{\eta}\right)-\varepsilon \int_{0}^{T} \log \left(u_{\eta}\right)
$$

and hence

$$
\limsup _{\varepsilon \rightarrow 0} F_{\varepsilon}\left(u_{\varepsilon}^{*}\right) \leq F\left(u_{\eta}\right) \leq \eta F\left(u^{*}\right)+(1-\eta) F\left(u_{0}\right),
$$

hence, with $\eta$ going to 1 , we have

$$
\limsup _{\varepsilon \downarrow 0} F_{\varepsilon}\left(u_{\varepsilon}^{*}\right) \leq F\left(u^{*}\right) .
$$

On the other hand, using lemma 1 and the fact that $u^{*}$ is optimal for $F$, the following inequality holds

$$
F_{\varepsilon}\left(u_{\varepsilon}^{*}\right) \geq\left(1-\frac{2 \varepsilon}{N}\right) F\left(u_{\varepsilon}^{*}\right)+m \varepsilon \geq\left(1-\frac{2 \varepsilon}{N}\right) F\left(u^{*}\right)+m \varepsilon,
$$

implying

$$
\liminf _{\varepsilon \rightarrow 0} F_{\varepsilon}\left(u_{\varepsilon}^{*}\right) \geq F\left(u^{*}\right)
$$

This proves (ii). 
We have already proved that the set $\hat{U}_{a d}^{1}$ defined in (6) is bounded, and hence, $\left(u_{\varepsilon}^{*}\right)_{\varepsilon>0}$ has a weakly convergent subsequence $u_{\varepsilon_{n}}^{*} \rightarrow v$. Since $U_{a d}$ is weakly closed, we have that $v \in U_{a d}$. By weak lower semi continuity of $F$, we get

$$
F(v) \leq \liminf _{\varepsilon_{n} \rightarrow 0} F\left(u_{\varepsilon_{n}}^{*}\right)=F\left(u^{*}\right),
$$

and hence, $v=u^{*}$. This proves that $u^{*}$ is the weak limit of $u_{\varepsilon}$. By standard compactness arguments, this implies (iii).

Finally, since $F$ is w.l.s.c., we have that $F\left(u^{*}\right) \leq \lim \inf F\left(\left(u_{\varepsilon}^{*}\right)\right.$. The inequality cannot be strict, since otherwise we would get a contradiction using the points $u_{\eta}$ constructed at the beginning of the proof. It follows that $\left\|u_{\varepsilon}^{*}-\hat{u}\right\| \longrightarrow\left\|u^{*}-\hat{u}\right\|$ in $L^{2}\left(0, T, \mathbb{R}^{m}\right)$, which combined with the weak convergence of $u_{\varepsilon}^{*}$ implies (i).

\section{Extension to a class of nonlinear optimal control prob- lems}

\subsection{Setting of the problem.}

Let us now generalize the results of the previous section to the case when the control enters linearly in the state equation and in a quadratic way in the cost function. Consider the state equation

$$
\left\{\begin{array}{l}
\dot{y}(t)=A(y(t))+B(y(t)) u(t), \quad t \in[0, T] \\
y(0)=y_{0}
\end{array}\right.
$$

and the associated optimal control problem

$$
\left\{\begin{array}{l}
\operatorname{Min}_{u} J(y, u)=\int_{0}^{T} \ell(y(t), u(t)) d t+\Phi(y(T)) \\
\text { subject to (11) and } u \in U_{a d} .
\end{array}\right.
$$

Here again $U_{a d}$ represents bound constraints on the control, but we assume now the bound to be finite, i.e.,

$$
U_{a d}=\left\{u \in L^{\infty}\left(0, T ; \mathbb{R}^{m}\right) ; a_{i} \leq u_{i}(t) \leq b_{i} i=1, \ldots, m, \text { a.e. } t \in(0, T)\right\},
$$

with $a$ and $b$ in $\mathbb{R}^{m}$. We make the following assumptions on the data:

(A1) The function $\ell: \mathbb{R}^{n+m} \rightarrow \mathbb{R}^{n}$ is twice continuously differentiable, and convex with respect to its second argument.

(A2) The mappings $A: \mathbb{R}^{n} \rightarrow \mathbb{R}^{n}$ and $B: \mathbb{R}^{n} \rightarrow \mathbb{R}^{n \times m}$ are of class $C^{2}, A$ has a linear growth, and $B$ is bounded, i.e., there exists constants $\bar{A}$ and $\bar{B}$ such that

$$
\|A(x)\| \leq \bar{A}(1+\|x\|) ; \quad\|B(x)\| \leq \bar{B}, \quad \text { for all } \quad x \in \mathbb{R} .
$$

INRIA 
We recall the following classical result.

Proposition 3 (i) With each $u \in U_{a d}$ is associated a unique state $y_{u} \in H^{1}\left(0, T ; \mathbb{R}^{n}\right)$ solution of (11), and the set of such states is bounded: there exists $K>0$ such that

$$
\left\|y_{u}\right\|_{\infty} \leq K, \quad \text { whenever } u \in U_{a d}
$$

(ii) If the sequence $\left(u_{n}\right)$ weakly converges in $L^{2}\left(0, T ; \mathbb{R}^{m}\right)$ to $\bar{u}$, then the corresponding sequence of states weakly converges in $H^{1}\left(0, T ; \mathbb{R}^{n}\right)$ to $y_{\bar{u}}$.

(iii) There exists $C>0$, such that, denoting by $\|\cdot\|$ the $L^{1}$ norm

$$
\left\|y_{u}-y_{v}\right\|_{\infty} \leq C\|u-v\|_{1}, \text { for all } u, v \in U_{a d} .
$$

Proof. Point (i) is an easy consequence of the definition of $U_{a d}$ and of the hypotheses made on $A(\cdot)$ and $B(\cdot)$. Let us prove (ii). Due to the compact embedding in $H^{1}\left(0, T ; \mathbb{R}^{n}\right)$ into $C\left(0, T ; \mathbb{R}^{n}\right)$, the state sequence $\left(y_{n}\right)$, that by $(14)$ is bounded in $H^{1}\left(0, T ; \mathbb{R}^{n}\right)$, has a uniformly convergent subsequence to some $\bar{y} \in C\left(0, T ; \mathbb{R}^{n}\right)$. Extracting a subsequence if necessary, we have that $\bar{y}$ is a weak limit of $\left(y_{n}\right)$ in $H^{1}\left(0, T ; \mathbb{R}^{n}\right)$. This allows to pass to the limit in the state equation, showing that $\bar{y}=y_{\bar{u}}$. It follows that the entire sequence $\left(y_{n}\right)$ converges to $y_{\bar{u}}$, as was to be shown.

(iii) Let $u$ and $v$ belong to $U_{a d}$, and denote $w:=u-v, z:=y_{u}-y_{v}$. By hypothesis (A2) and (14), $A$ and $B$ are Lipschitz over the set of states associated with feasible controls, and hence, denoting by $L$ an upper bound of the Lipschitz constants,

$$
\begin{aligned}
|\dot{z}(t)| & \leq\left|A\left(y_{u}(t)\right)-A\left(y_{v}(t)\right)\right|+\left|B\left(y_{u}(t)\right) u(t)-B\left(y_{v}(t)\right) v(t)\right| \\
& \leq L|z(t)|+\left|\left(B\left(y_{u}(t)\right)-B\left(y_{v}(t)\right)\right) u(t)+B\left(y_{v}(t)\right)(u(t)-v(t))\right| \\
& \leq L|z(t)|(1+|u(t)|)+\bar{B}|w(t)|,
\end{aligned}
$$

from which the result follows by Gronwall's lemma.

We can write problem $(P)$ under the form

$$
\operatorname{Min}_{u \in U_{a d}} F(u),
$$

where $F(u)=J\left(y_{u}, u\right)$ is a real function over $L^{2}\left(0, T ; \mathbb{R}^{m}\right)$.

The Hamiltonian function of the problem is

$$
H(y, p, u)=\ell(y, u)+p^{T}(A(y)+B(y) u),
$$

and the costate $p_{u}$ associated with a control $u$ is defined by the backwards equation

$$
\dot{p}_{u}(t)=-H_{y}\left(y_{u}(t), p_{u}(t), u(t)\right), \quad \text { a.e. } t \in(0, T) ; \quad p_{u}(T)=0 .
$$

Lemma 2 Under assumption (A1) and (A2), the cost function $F$ is weakly sequentially lower semi continuous over $U_{a d}$.

$\mathrm{RR} \mathrm{n}^{\circ} 4237$ 
Proof. Let $\left(u_{n}\right)$ converge weakly towards $u$ in $U_{a d}$. The sequence $y_{n}$ of associated states converges uniformly to $y_{u}$. By proposition 3(ii), all feasible controls and associated states remain in a compact set, over which $\ell$ is uniformly continuous. It follows that $\left[\ell\left(y_{u_{n}}(t), u_{n}(t)\right)-\ell\left(y_{u}(t), u_{n}(t)\right)\right] \longrightarrow 0$ uniformly. Therefore,

$$
\liminf _{n \rightarrow \infty} F\left(y_{u_{n}}, u_{n}\right)=\liminf _{n \rightarrow \infty} F\left(y_{u}, u_{n}\right) \geq F\left(y_{u}, u\right)
$$

since $F\left(y_{u},.\right)$ is convex continuous, and hence weakly lower semi continuous. The conclusion follows.

We assume that problem $(P)$ satisfies the following assumption.

(A3) Problem $(P)$ has a unique solution $u^{*}$, that satisfies the local quadratic growth property: there exists $r>0$ such that

$$
F(u)-F\left(u^{*}\right) \geq \beta\left\|u-u^{*}\right\|_{2}^{2}, \quad \forall u \in U_{a d}, \quad\left\|u-u^{*}\right\|_{L^{2}\left(0, T, \mathbb{R}^{m}\right)} \leq r .
$$

This condition, when the data of problem $(P)$ (i.e., $A, B, \ell$ and $\Phi$ ) are of class $C^{2}$, is equivalent to the second order sufficient condition (see Bonnans and Shapiro [6]:

$$
\left\{\begin{array}{l}
D^{2} F\left(u^{*}\right)(h, h) \geq \beta\|h\|_{2}^{2} \quad \forall h \in L^{2}(0, T) \quad \text { such that } \\
\left\{\begin{array}{l}
D F\left(u^{*}\right) h=0 \\
h_{i} \geq 0 \text { a.e. on }\left\{t \in[0, T] / u_{i}(t)=a_{i}\right\} \\
h_{i} \leq 0 \text { a.e. on }\left\{t \in[0, T] / u_{i}(t)=b_{i}\right\} .
\end{array}\right.
\end{array}\right.
$$

\subsection{The penalized problem.}

We now introduce the logarithmic penalty in the spirit of the previous section. The localized penalized problem is:

$$
\operatorname{Min}_{u \in B\left(u^{*}, r\right)} F_{\varepsilon}(u):=F(u)-\varepsilon \sum_{i=1}^{m} \int_{0}^{T}\left[\log \left(b_{i}-u_{i}(t)\right)+\log \left(u_{i}(t)-a_{i}\right)\right] d t
$$

The discussion on the domain of definition of $F_{\varepsilon}$ is similar to the one in the previous section; i.e.,

$$
\operatorname{dom}\left(F_{\varepsilon}\right)=\left\{u \in U_{a d} ; \log \left(b_{i}-u_{i}(t)\right) \text { and } \log \left(u_{i}(t)-a_{i}\right) \text { are integrable }\right\}
$$

is a convex, but not closed subset of $U_{a d}$.

Lemma 3 For every $\varepsilon>0$, problem $\left(P_{\varepsilon}\right)$ has a finite value.

Proof. The feasible controls and associated states being uniformly bounded, and $\ell$ is continuous, we have that $F$ is lower bounded on $U_{a d}$. On the other hand,

$$
-\int_{0}^{T}[\log (u(t)-a)+\log (b-u(t))] d t \geq-2 T \log (b-a),
$$


and hence,

$$
F_{\varepsilon}(u) \geq F(u)-2 \varepsilon T \log (b-a), \quad \text { for all } \quad u \in U_{a d} .
$$

The lemma follows.

Theorem 2 For every $\varepsilon>0$, problem $\left(P_{\varepsilon}\right)$ has (at least one) a solution $u_{\varepsilon}^{*}$, and there exists $\alpha(\varepsilon)>0$, with $\liminf \varepsilon^{-1} \alpha(\varepsilon)>0$, such that

$$
\left.a+\alpha(\varepsilon) \leq u_{\varepsilon}^{*} \leq b-\alpha(\varepsilon)\right\}, \quad \alpha(\varepsilon)>0 .
$$

Moreover $u_{\varepsilon}^{*}$ is continuous over $[0, T]$.

Proof. It is enough to give the proof when $u$ is scalar. Let $u \in U_{a d}^{1}$ be such that infess $u=0$. Given $\alpha>0$, let $v$ be defined by (7). Then

$$
F_{\varepsilon}(v)-F_{\varepsilon}(u)=\Delta_{1}+\Delta_{2}+\varepsilon \Delta_{3},
$$

with

$$
\begin{aligned}
\Delta_{1} & :=\int_{0}^{T}\left[\ell\left(y_{v}(t), v(t)\right)-\ell\left(y_{v}(t), u(t)\right)\right] d t, \\
\Delta_{2}: & =\int_{0}^{T}\left[\ell\left(y_{v}(t), u(t)\right)-\ell\left(y_{u}(t), u(t)\right)\right] d t, \\
\Delta_{3} & :=\int_{0}^{T} \log (u(t) / v(t)) d t .
\end{aligned}
$$

For $\Delta_{3}$ we have the estimate (8). Also, denoting by $L_{\ell}$ a Lipschitz constant of $\ell$ over all possible admissible controls and associated states,

$$
\Delta_{1} \leq L_{\ell} \int_{0}^{T}|u(t)-v(t)| d t \leq 3 \alpha L_{\ell} \operatorname{mes}(u \leq \alpha) .
$$

Using the constant $C$ defined in proposition 3 (ii), obtain

$$
\Delta_{2} \leq L_{\ell} \int_{0}^{T}\left|y_{v}(t)-y_{u}(t)\right| d t \leq C L_{\ell} \int_{0}^{T}|u(t)-v(t)| d t
$$

and hence, combining these results,

$$
F_{\varepsilon}(v)-F_{\varepsilon}(u) \leq\left[3(1+C) \alpha L_{\ell}-\varepsilon \log 2\right] \operatorname{mes}(u \leq \alpha) .
$$

Taking

$$
\alpha=\alpha(\varepsilon)=\frac{\log 2}{3(1+C) \alpha L_{\ell}},
$$

obtain that $F_{\varepsilon}(v) \leq F_{\varepsilon}(u)$. By the same arguments as for linear quadratic control problems, we may therefore construct a minimizing sequence with value in $[a+\alpha(\varepsilon), b-\alpha(\varepsilon)]$. Hence,

$\mathrm{RR} \mathrm{n}^{\circ} 4237$ 
the cost function being weakly l.c.s on this set, this proves that the penalized problem has at least a solution.

It remains now to check continuity of $u_{\varepsilon}^{*}$. The Hamiltonian function of the penalized problem is

$$
H(y, p, u)=H(y, p, u)-\varepsilon\left(\log \left(b_{i}-u_{i}(t)\right)+\log \left(u_{i}(t)-a_{i}\right)\right) .
$$

Hypothesis (A1) implies that $H_{u u}(y(t), u(t), p(t))>0$, for all $t \in[0, T]$. Since the penalty function has everywhere a positive definite Hessian, it follows that

$$
H_{u u}^{\varepsilon}(y(t), u(t), p(t))>0 .
$$

Hence, applying the implicit function theorem to the necessary optimality condition

$$
H_{u}^{\varepsilon}(y(t), u(t), p(t))=0, \quad t \in[0, T],
$$

and using the fact that the state and costate are continuous functions, we obtain that the control is continuous. But then the state, and hence the costate, are of class $C^{1}$. It follows then that the control is itself of class $C^{1}$.

The costate equation for the penalized problem is

$$
-\dot{p}=H_{y}^{\varepsilon}(y(t), u(t), p(t)), \quad t \in[0, T] .
$$

The convergence result is the following

Proposition 4 Let $u_{\varepsilon}^{*}$ be solutions of $\left(P_{\varepsilon}\right)$, and denote by $\left(y_{\varepsilon}^{*}\right)$ and $\left(p_{\varepsilon}^{*}\right)$ the associated states and costates. Then the following relations hold:

(i) The controls $u_{\varepsilon}^{*}$ converge to $u^{*}$ in $L^{2}\left(0, T ; \mathbb{R}^{m}\right)$.

(ii) One has $F_{\varepsilon}\left(u_{\varepsilon}^{*}\right) \longrightarrow F\left(u^{*}\right)$.

(iii) The convergence of $\left(y_{\varepsilon}^{*}\right)\left(\right.$ resp. $\left.\left(p_{\varepsilon}^{*}\right)\right)$ towards $y^{*}$ (resp. $\left.p^{*}\right)$ holds.

Proof. Again, assume that the control is scalar for the sake of simplicity. Let $u_{0}$ be such that infess $u_{0}>a$ and supess $u_{0}<b$, and set $u_{\eta}=u^{*}+\eta\left(u_{0}-u^{*}\right)$. Then, for a given $\eta \in(0,1)$, we have that

$$
F_{\varepsilon}\left(u_{\varepsilon}^{*}\right) \leq F_{\varepsilon}\left(u_{\eta}^{*}\right)=F\left(u_{\eta}^{*}\right)+O(\varepsilon) .
$$

Passing to the limit when $\varepsilon \downarrow 0$, and then when $\eta \downarrow 0$, we obtain that

$$
\limsup _{\varepsilon \downarrow 0} F_{\varepsilon}\left(u_{\varepsilon}^{*}\right) \leq F\left(u^{*}\right) .
$$

The converse inequality is an immediate consequence of (15). Point (ii) follows. 
Combine point (ii) and (15) with $u=u_{\varepsilon}$, to obtain that $\lim \sup _{\varepsilon \downarrow 0} F_{\varepsilon}\left(u_{\varepsilon}^{*}\right) \leq F\left(u^{*}\right)$. Since $F$ is weakly l.s.c., it follows that $u_{\varepsilon}$ weakly converges to $u^{*}$. At the same time, since $y_{\varepsilon}^{*} \longrightarrow y^{*}$ uniformly, we have that

$$
\left.\left.\int_{0}^{T}\left[\ell\left(y_{\varepsilon}^{*}, u_{\varepsilon}^{*}\right)\right)-\ell\left(y_{\varepsilon}^{*}, u_{\varepsilon}^{*}\right)\right)\right] d t \longrightarrow 0,
$$

and hence,

$$
F\left(u_{\varepsilon}^{*}\right) \leq \liminf _{\varepsilon} \int_{0}^{T} \ell\left(y^{*}(t), u_{\varepsilon}^{*}(t)\right) d t .
$$

Again the strict inequality is not possible since we would obtain a contradiction by using

the controls $u_{\eta}$ constructed above. Our hypotheses imply that $u \longrightarrow \int_{0}^{T} \ell\left(y^{*}(t), u(t)\right) d t$ is strongly convex over $U_{a d}$, and therefore, any minimizing sequence is strongly convergent, which proves (i).

\section{Some numerical applications}

We have solved numerically some examples by using the OCS code [5], which is an implementation of the shooting algorithm for unconstrained problems. Let us recall briefly the principles of the shooting method. Given an optimal control problem of the following form,

$$
\left\{\begin{array}{l}
\min _{u} \int_{0}^{T} \ell(y(t), u(t)) d t+\phi(y(T)) \\
\dot{y}(t)=f(y(t), u(t)), \quad t \in[0, T], \quad y(0)=y_{0},
\end{array}\right.
$$

the goal of the shooting method is to solve the associated optimality system

$$
\begin{gathered}
\left\{\begin{array}{lll}
\dot{y}(t) & =f(y(t), u(t)), & t \in[0, T], \\
\dot{p}(t) & =-D_{y} H(y(t), p(t), u(t)), & t \in[0, T], \\
0 & =D_{u} H(y(t), p(t), u(t)), & t \in[0, T]
\end{array}\right. \\
\\
y(0)=y_{0}, \quad p(T)=D \phi(y(T))
\end{gathered}
$$

where again $H$ is the Hamiltonian of the system. Then we define what we call the shooting function $\Theta: p_{0} \longmapsto p_{T}$ which, with a value $p_{0}$ of the initial state associates the value $p(T)-D \phi(y(T))$, where $(y, p, u)$ is solution of $(16)$. We have that $(y, p, u)$ is a solution of the optimality system (16)-(17) iff $\Theta(p(0))=0$. OCS solves the algebraic-differential system (16) using the code dassl, see Petzold [15]. Newton's method with line search and exact computation of the Jacobian is applied to the shooting function $\Theta$. As usual, finding a good initial point for Newton's method may be a hard task. We illustrate the mixed method with shooting and logarithmic penalty this with some examples. 


\subsection{Flight stabilization under constraints.}

Let us first consider the following flight stabilization problem consisting in stabilizing a flying object which is falling. The state equations are a simplified model of equations for the flight of an aircraft in a vertical plane. The problem is stated the following way:

$$
\underset{u \in U_{a d}}{\operatorname{Minimize}} \frac{1}{2} \int_{0}^{T}(u(t)-\alpha)^{2} d t+\frac{1}{2} \int_{0}^{T} \gamma(t)^{2} d t
$$

with the dynamics

$$
\left\{\begin{aligned}
\dot{v} & =-c D v^{2}-g \sin (\gamma), & & v(0)=v_{0}, \\
\dot{\gamma} & =c L v u-\frac{g \cos (\gamma)}{v}, & & \gamma(0)=\gamma_{0}, \\
\dot{h} & =v \sin (\gamma), & & h(0)=h_{0},
\end{aligned}\right.
$$

where $c_{D}$ et $c_{L}$ aerodynamical constants and $v$ is the speed, $\gamma$ the flight-path angle, $h$ the altitude and $u$, the control, is the angle of attack. The horizon is $T=50$ seconds, $\alpha=\frac{1}{2}$ and the initial value of the state is:

$$
v_{0}=0.36, \quad \gamma_{0}=-1.14, \quad h_{0}=15 .
$$

The constraint on the control is

$$
U_{a d}=\{u, u(t) \geq \beta \quad \forall t \in[0, T]\},
$$

with $\beta=0.55$. We also add final constraints on the flight-path angle

$$
\gamma(T)=-\gamma(0) .
$$

We give the constrained solution, computed with a penalizing parameter equal to $10^{-4}$. The norm of the shooting function value after 20 Newton steps is $10^{-11}$. See the computed trajectory in figure 4.2 .

\subsection{Stabilization of an oscillating system.}

The second example deals with stabilizating an oscillating system whose uncontrolled dynamic is defined by

$$
m \ddot{x}(t)=k x+A\left(\sin \left(\omega_{1} t\right)+\sin \left(\omega_{2} t\right)\right)
$$

where $k$ is the stiffness coefficient, $m$ is the mass and $\omega=\sqrt{\frac{k}{m}}$ is the resonance frequency of the system and $\omega_{1}=\omega+\frac{\omega}{2}, w_{2}=\omega-\frac{\omega}{2}$.

The control problem is stated:

INRIA 


$$
\text { Minimize } \frac{1}{2} \int_{0}^{4 \pi} x(t)^{2}+\frac{1}{2} \int_{0}^{4 \pi} u(t)^{2}
$$

with the dynamics

$$
\ddot{x}(t)=-x(t)+0.5\left(\sin \left(\omega_{1} t\right)+\sin \left(\omega_{2} t\right)\right)+u(t),
$$

where we don't specify $x(0)$ et $\dot{x}(0)$ which are free. We set $\omega_{1}=\frac{3}{2}$ and $\omega_{2}=\frac{1}{2}$. The constraints on the control are

$$
U_{a d}=\{u,|u(t)| \geq \beta \quad \forall t \in[0, T]\},
$$

where $\beta=0.3$, and we also add a periodicity constraint

$$
x(0)=x(4 \pi) \text { and } \dot{x}(0)=\dot{x}(4 \pi) .
$$

In this problem, for the Shooting, we have in fact 3 state variables: the state variable $x$, its time derivative $v$ and time $t$ since the differential equation is nonautonomous. We show both unconstrained an constrained optimal solutions in figures (2) and (3). For the constrained case, we have set the penalty parameter equal to $10^{-5}$. After 4 Newton steps, the value of the shooting function is around $10^{-6}$.

$\mathrm{RR} \mathrm{n}^{\circ} 4237$ 


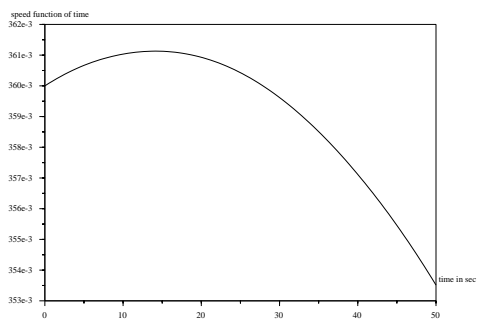

Speed.

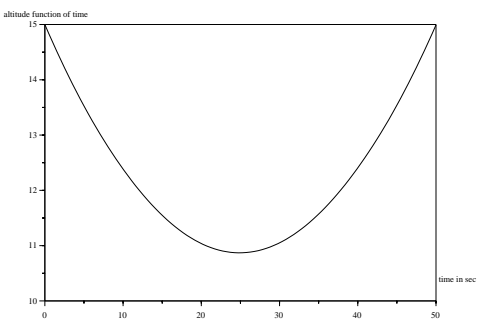

Altitude.

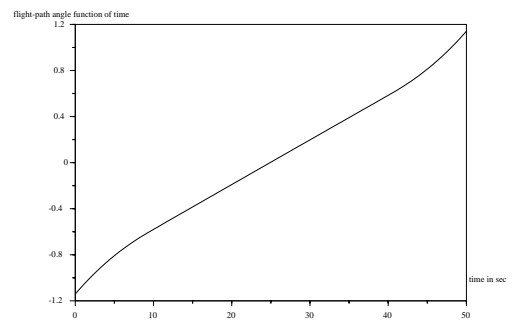

Flight-path angle.

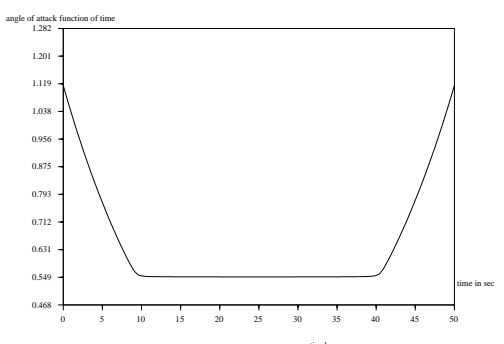

Control.

Figure 2: Flight stabilization under constraint.
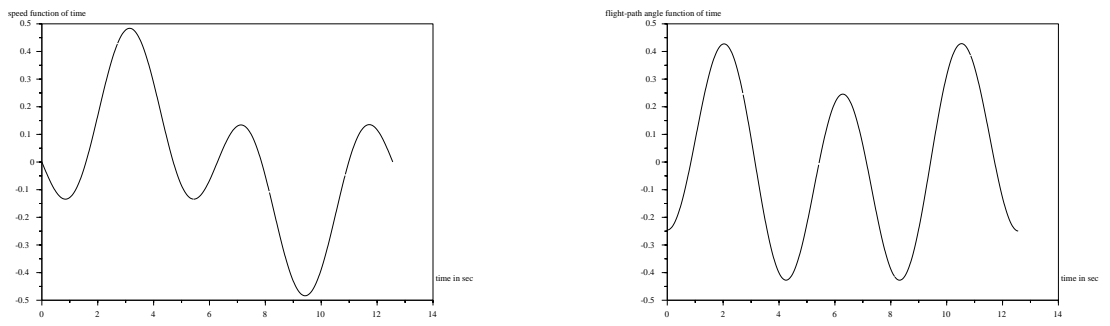

Figure 3: The state and its time derivative. 


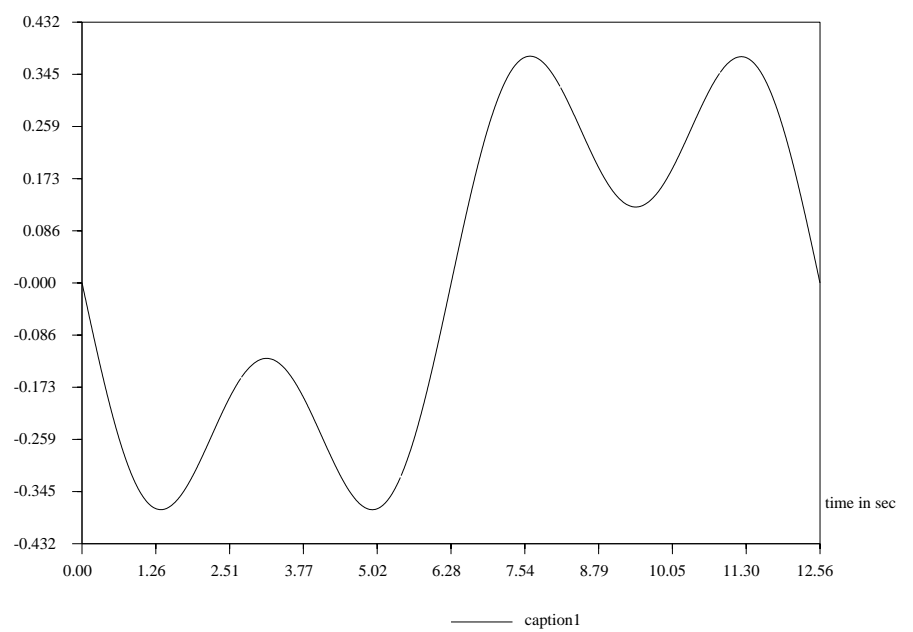

Figure 4: The optimal control without constraint.
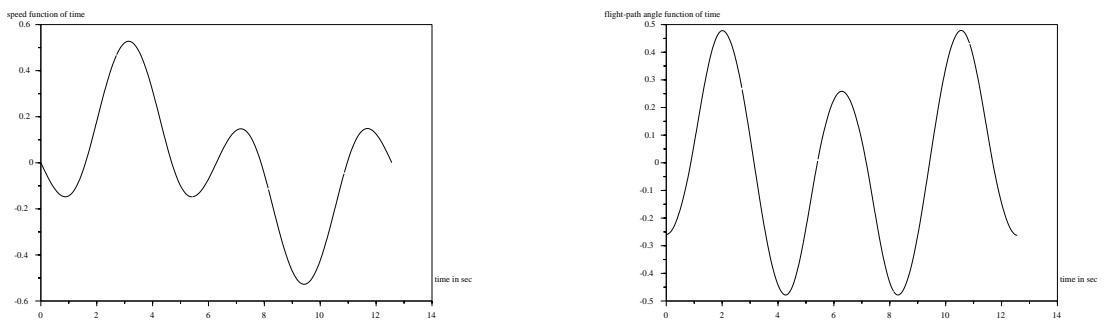

Figure 5: The state and its time derivative.

$\mathrm{RR} \mathrm{n}^{\circ} 4237$ 


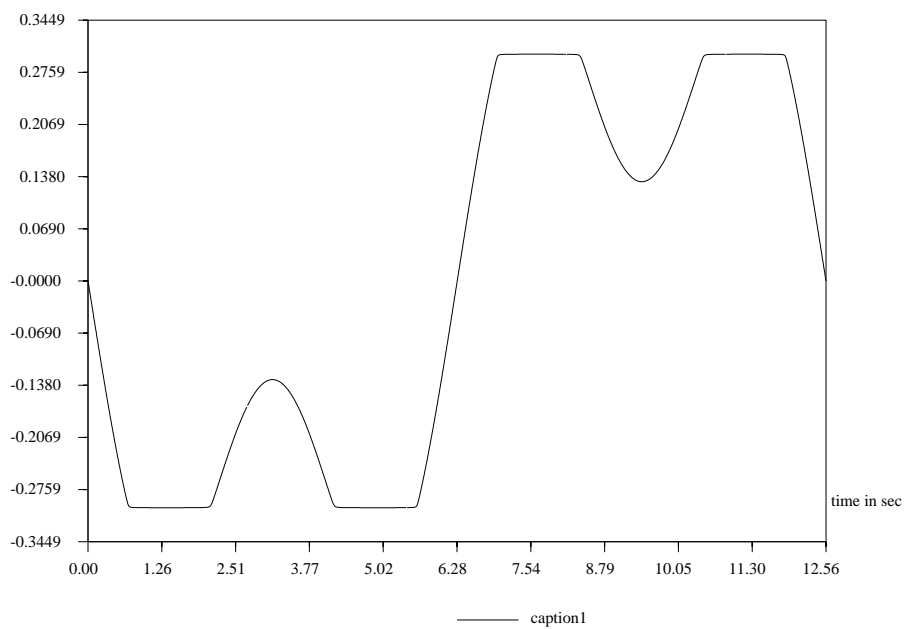

Figure 6: The optimal control under constraints.

\section{References}

[1] J.T. Betts. Survey of numerical methods for trajectory optimization. AIAA J. of Guidance, Control and Dynamics, 21:193-207, 1998.

[2] J.T. Betts and W.P. Huffman. Application of sparse nonliner programming to trajectory optimization. AIAA J. of Guidance, Control and Dynamics, 15:198-206, 1992.

[3] J.F. Bonnans, J.Ch. Gilbert, C. Lemaréchal, and C. Sagastizábal. Optimisation Numérique: aspects théoriques et pratiques, volume 27 of Séries Mathématiques et Applications. Springer-Verlag, Paris, 1997.

[4] J.F. Bonnans and G. Launay. Large scale direct optimal control applied to a re-entry problem. AIAA J. of Guidance, Control and Dynamics, 21:996-1000, 1998.

[5] J.F. Bonnans and S. Maurin. An implementation of the shooting algorithm for solving optimal control problems. Technical Report RT-0240, INRIA, 2000.

[6] J.F. Bonnans and A. Shapiro. Perturbation analysis of optimization problems. SpringerVerlag, New York, 2000.

[7] R. Bulirsch, E. Nerz, H. J. Pesch, and O. von Stryk. Combining direct and indirect methods in optimal control: range maximization of a hang glider, pages 273-288. Birkhäuser, Basel, 1993. 
[8] X. Chen, Z. Nashed, and L.Q. Qi. Smoothing methods and semismooth methods for nondifferentiable operator equations. SIAM J. on Numerical Analysis, 38:1200-1216 (electronic), 2000.

[9] H.-J. Diekhoff, P. Lory, H.J. Oberle, H.-J. Pesch, P. Rentrop, and R. Seydel. Comparing routines for the numerical solution of initial value problems of ordinary differential equations in multiple shooting. Numerische Mathematik, 27:449-469, 1977.

[10] L.C.W. Dixon and M.C. Bartholomew-Biggs. Adjoint-control transformations for solving pratical optimal control problems. Optimal Control, Applications and Methods, $2: 365-381,1981$.

[11] A.V. Fiacco and G.P. McCormick. Nonlinear Programming: Sequential Unconstrained Minimization Techniques. Wiley, New York, 1968.

[12] G. Fraser-Andrews. Shooting method for the numerical solution of optimal control problems with bounded state variables. J. of Optimization Theory and Applications, 89:351-372, 1996.

[13] J.M. Martínez and L.Q. Qi. Inexact Newton methods for solving nonsmooth equations. J. of Computational and Applied Mathematics, 60:127-145, 1995.

[14] H.J. Pesch. Real-time computation of feedback controls for constrained optimal control problems II: a correction method based on multiple shooting. Optimal Control, Applications and Methods, 10:147-171, 1989.

[15] L.R. Petzold. A description of DASSL: a differential/algebraic system solver. In Scientific computing (Montreal, Quebec, 1982), pages 65-68. IMACS, New Brunswick, NJ, 1983.

[16] J. Stoer and R. Bulirsch. Introduction to Numerical Analysis. Springer-Verlag, NewYork Heidelberg Berlin, 1993.

[17] T. Terlaky, editor. Interior Point Methods of Mathematical Programming. Kluwer, Boston, 1996.

[18] S.J. Wright. Primal-dual interior-point methods. SIAM, Philadelphia, 1996.

$\mathrm{RR} \mathrm{n}^{\circ} 4237$ 


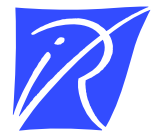

Unité de recherche INRIA Rocquencourt Domaine de Voluceau - Rocquencourt - BP 105 - 78153 Le Chesnay Cedex (France)

Unité de recherche INRIA Lorraine : LORIA, Technopôle de Nancy-Brabois - Campus scientifique 615, rue du Jardin Botanique - BP 101 - 54602 Villers-lès-Nancy Cedex (France)

Unité de recherche INRIA Rennes : IRISA, Campus universitaire de Beaulieu - 35042 Rennes Cedex (France)

Unité de recherche INRIA Rhône-Alpes : 655, avenue de l'Europe - 38330 Montbonnot-St-Martin (France)

Unité de recherche INRIA Sophia Antipolis : 2004, route des Lucioles - BP 93 - 06902 Sophia Antipolis Cedex (France)

INRIA - Domaine de Voluceau - Rocquencourt, BP 105 - 78153 Le Chesnay Cedex (France)

http://www.inria.fr

ISSN 0249-6399 